\title{
Effect of Orally Administered Glutathione-Montmorillonite Hybrid Systems on Tissue Distribution
}

\author{
Miri Baek and Soo-Jin Choi \\ Department of Food Science and Technology, Seoul Women's University, Seoul 139-774, Republic of Korea \\ Correspondence should be addressed to Soo-Jin Choi, sjchoi@swu.ac.kr \\ Received 13 February 2012; Accepted 12 April 2012 \\ Academic Editor: Haifeng Chen
}

Copyright (๑) 2012 M. Baek and S.-J. Choi. This is an open access article distributed under the Creative Commons Attribution License, which permits unrestricted use, distribution, and reproduction in any medium, provided the original work is properly cited.

\begin{abstract}
An ubiquitous tripeptide, glutathione (GSH), is assigned a role in detoxification, activation of immune system, intermediary metabolism, transport, and protection of cells against free radicals or reactive oxygen species. However, instability of orally administered GSH in gastrointestinal (GI) tract leads to low absorption and low bioavailability in tissues. In this study, we attempted to synthesize GSH-montmorillonite (MMT) hybrid systems by intercalating GSH into the interlayers of a cationic clay delivery carrier, MMT, to improve GSH bioavailability at the systemic level. Polymer coating of the hybrid with polyvinylacetal diethylaminoacetate (AEA) was further performed to obtain better stability. Synthetic condition of both GSH-MMT and AEAGSH-MMT hybrids was optimized, and then GSH-delivery efficiency was evaluated in various organs after oral administration in normal as well as GSH-deficient mice. The present GSH-MMT hybrids remarkably enhanced GSH concentration in the plasma, heart, kidney, and liver, especially when AEA-GSH-MMT hybrid was administered under GSH-deficient condition. Moreover, both hybrids did not induce acute oral toxicity up to $2000 \mathrm{mg} / \mathrm{kg}$, suggesting their great potential for pharmaceutical application.
\end{abstract}

\section{Introduction}

GSH (L-glutamyl-L-cysteinylglycine) is the most abundant low molecular thiol compound widely present in living plants and animals [1]. GSH is an important substance involved in a wide range of biological functions such as metabolism, catalysis, amino acid transport, cysteinereservoir, detoxification, and removal of free radicals [2, $3]$. It also plays a role in many cellular events by modulating redox-regulated cellular signal transduction and regulating cell proliferation, protein/gene expression, and immune response via thiol-disulfide exchange reaction [46]. It was also suggested that GSH may be implicated in cancer prevention [7]. Thus, GSH deficiency induces oxidative stress, which is implicated in the pathogenesis of many diseases including Alzheimer', Parkinson's, acute respiratory, ocular and liver diseases, AIDS, and heart attack. In particular, GSH depletion was reported to be closely associated with increased virus replication in HIV-positive patients [8-10]. GSH can be absorbed intact from the intestinal lumen, taken up by enterocytes and finally released into the blood stream [11]. However, most of orally ingested GSH tends to be rapidly degraded to free amino acids by both chemical and enzymatic hydrolysis such as $\gamma$-glutamyltranspeptidase and $\gamma$-glutamyl-cyclotransferase, leading to low absorption rate during GI transit $[12,13]$. Moreover, the thiol group of GSH can be easily oxidized both enzymatically and nonenzymatically under intestinal condition, forming glutathione disulfide (GSSG) which is devoid of antioxidant activity [14].

The aim of this study was, therefore, to enhance the bioavailability of oral GSH in vivo by developing a novel delivery system in which GSH was intercalated into the layers of a cationic clay, MMT. It is a type of smectite clay mineral composed of structural units of silica tetrahedral sheets with a central alumina octahedral sheet. Its unique structure gives rise to having large specific surface area, adsorption ability, cation exchange capacity (CEC), and adhesive ability, which are attractive properties as a delivery carrier, leading to intercalate labile molecule into the interlayer spaces via ion 


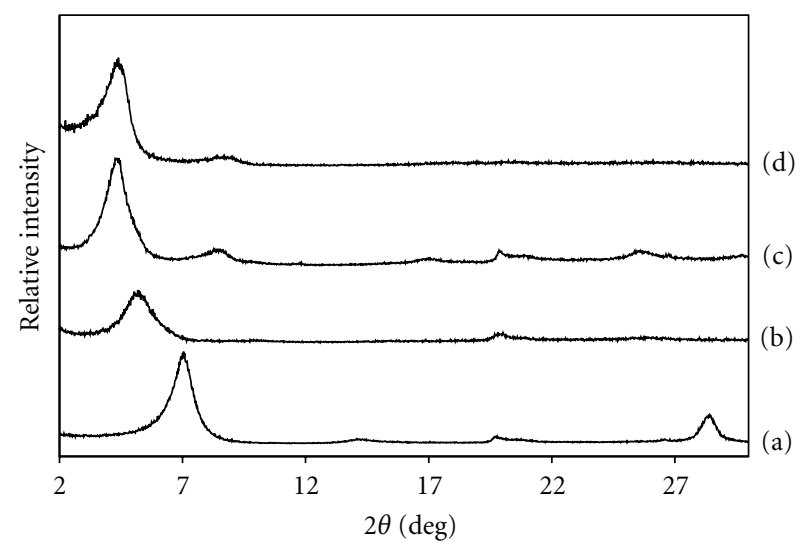

(A)

(a)
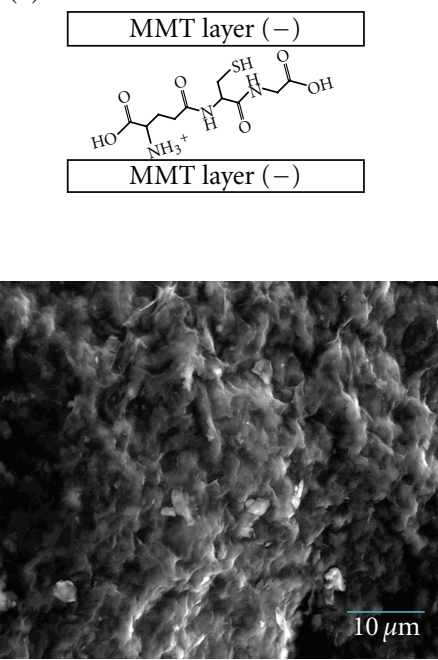
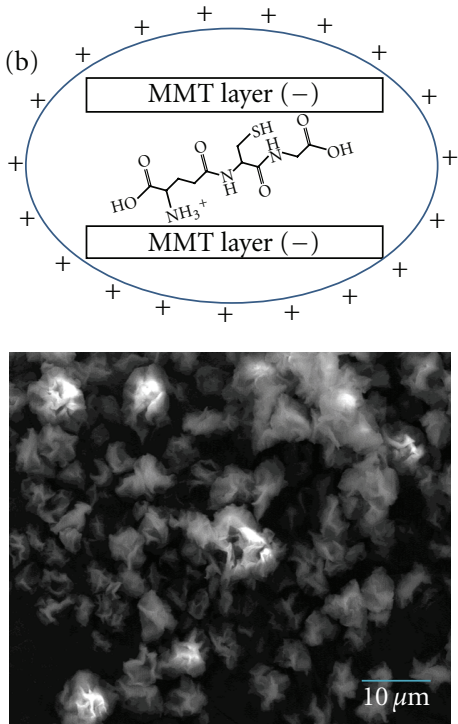

(B)

Figure 1: PXRD patterns (A) of pristine MMT, (a) GSH-MMT hybrid (CEC1), (b) GSH-MMT hybrid (CEC2), and (c) GSH-MMT hybrid (CEC3). (d) Schematic diagram and SEM images (B) of GSH-MMT hybrid (a) and AEA-GSH-MMT hybrid (b).

exchange reaction [15]. It was also reported that MMT has low toxicity and favorable kinetic behaviors in terms of no accumulation into specific tissues and rapid clearance from the body [16]. In our previous study, we were successful to develop both GSH-MMT and AEA-GSH-MMT hybrid systems [17]. The latter was obtained by polymer coating of the former with positively charged AEA for better stability at the systemic level, demonstrating that the latter significantly enhanced pharmacokinetic behaviors of GSH in mice by enhancing absorption amount and prolonging circulation time [17].

In the present study, we optimized the synthetic condition and evaluated the efficacy of GSH-MMT and AEA-GSHMMT hybrids, focusing on tissue distribution in normal as well as GSH-deficient mice. Moreover, the acute oral toxicity of both hybrid systems was assessed in mice to provide practical information for their pharmaceutical application.

\section{Materials and Methods}

2.1. Materials. $\mathrm{Na}^{+}-\mathrm{MMT}$ with a CEC of $0.7-1.1$ mequiv./g and AEA were supplied by Sigma-Aldrich (St. Louis, Mo, USA) and Sankyo (Tokyo, Japan), respectively. GSH and all the other reagents of high purity were purchased from Sigma-Aldrich.

2.2. Preparation of GSH-MMT Hybrids. GSH-MMT hybrid was prepared as follow; $10 \mathrm{~g}$ of $\mathrm{Na}^{+}$-MMT was dispersed in $900 \mathrm{~mL}$ of deionized water and vigorously stirred for $4 \mathrm{~h}$ at room temperature. The various ratios of GSH compared with $10 \mathrm{~g}$ of MMT (1-, 2- and 3-fold of CEC) were dissolved in $100 \mathrm{~mL}$ of deionized water ( $\mathrm{pH}$ value adjusted to 2.0 with $\mathrm{HCl}$ ) and added to MMT suspension. The final $\mathrm{pH}$ of the GSH and MMT mixture solution was adjusted to 2.0 by addition of $\mathrm{HCl}$ solution. Thus, prepared suspension 


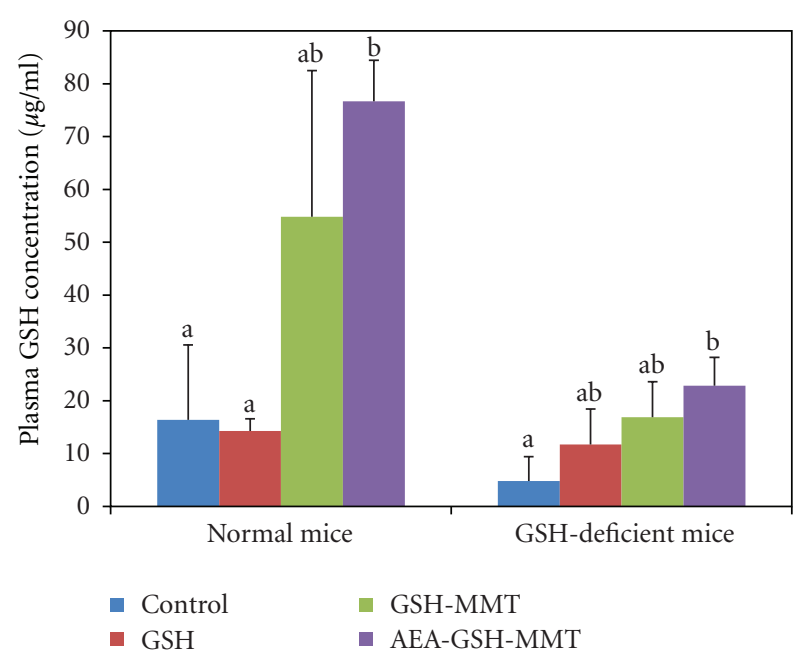

Figure 2: GSH concentration in the plasma at $1 \mathrm{~h}$ postoral administration of free GSH, GSH-MMT hybrid, or AEA-GSHMMT hybrid in normal as well as GSH-deficient mice. Different letters indicate a significant difference based on ANOVA and Tukey's test $(P<0.05)$.

was stirred at room temperature overnight and finally the resulting product was freeze-dried. To obtain polymer coated GSH-MMT hybrid, the prepared GSH-MMT hybrid was redispersed in $800 \mathrm{~mL}$ of ethanol solution and $10 \mathrm{~g}$ of AEA in $100 \mathrm{~mL}$ of methylene chloride (MC) was then added. The final AEA-GSH-MMT hybrid was spray dried (EYLA spray dryer SD-1000, Tokyo, Japan) under the following conditions: atomizing pressure, $130 \mathrm{kPa}$; blower speed, $0.30 \mathrm{~m}^{3} / \mathrm{min}$; inlet temperature, $80^{\circ} \mathrm{C}$; outlet temperature $40-50^{\circ} \mathrm{C}$.

2.3. Characterization of GSH-MMT Hybrids. The prepared GSH-MMT hybrids were characterized by powder X-ray diffraction (PXRD) using a diffractometer (Rigaku D/MAX RINT 2200-Ultima+, Japan) with Ni-filtered $\mathrm{CuK} \alpha$ radiation $(\lambda=1.5418 \AA$, voltage of $40 \mathrm{kV}$, a current of $30 \mathrm{~mA}$, and a scanning rate of $2 \% \mathrm{~min}$ ). The morphology of the hybrids was observed by scanning electron microscopy (SEM; MIRA FESEM, Teskan).

2.4. Animal and Diets. Male ICR mice, aged 5.5 weeks and weighing $24 \pm 1 \mathrm{~g}$, were purchased from the G-Bio (Seoul, Republic of Korea). The animals were housed in plastic lab animal cages in a ventilated room. The room was maintained at $20 \pm 2^{\circ} \mathrm{C}$ and $60 \pm 10 \%$ relative humidity with a $12 \mathrm{~h}$ light/dark cycle. Water and commercial laboratory complete food for mice were available ad libitum. They were acclimated to this environment for 7 days before treatment. GSHdeficient mouse model was induced by pretreatment with the GSH synthesis inhibitor, L-buthionine-S, R-sulfoximine (BSO, $20 \mathrm{mM}$ via drinking water), for 5 days [18]. All animal experiments were performed in compliance with the Animal and Ethnics Review Committee of the Seoul Women's University.
2.5. Plasma Concentration. The plasma concentration of GSH was analyzed after oral administration of GSH or the hybrids in normal and GSH-deficient mice, respectively. Each group of three mice was administrated via oral gavage with $100 \mathrm{mg} / \mathrm{kg}$ of free GSH or equivalent amount of the hybrids on the basis of GSH content from the hybrids. Another three mice group administered with $0.9 \%$ saline was used as a control group. The blood samples were collected via orbital sinus at $1 \mathrm{~h}$ postoral administration and then centrifuged at $3000 \mathrm{rpm}$ for $15 \mathrm{~min}$ at $4^{\circ} \mathrm{C}$ to obtain the plasma and stored at $-70^{\circ} \mathrm{C}$ before analysis. The proteins in the plasma were precipitated by adding 4 volumes of acetonitrile, followed by vortexing for $20 \mathrm{sec}$ and centrifuging at $10000 \mathrm{rpm}$ for $10 \mathrm{~min}$ at $4^{\circ} \mathrm{C}$. The supernatant was then filtered by a nylon membrane with a pore size of $0.45 \mu \mathrm{m}$ (Whatman, UK), and GSH concentration was then measured by high-performance liquid chromatography (HPLC) using an LC10-ADVP series (Shimadzu Co., Japan) on a Discovery RP-Amide 16 column $(150 \times 4.6 \mathrm{~mm}, 5 \mu \mathrm{m}$; Sigma, USA $)$. The mobile phase was $50 \mathrm{mM} \mathrm{NaClO} \mathrm{m}_{4} 0.1 \% \mathrm{H}_{3} \mathrm{PO}_{4}$, and flow rate was set to $1 \mathrm{~mL} / \mathrm{min}$. Column temperature was maintained at $40^{\circ} \mathrm{C}$, and detection of GSH was performed at $215 \mathrm{~nm}$ by UV detector [19].

2.6. Tissue Distribution. To evaluate delivery efficiency of GSH to organs, tissue samples such as brain, heart, kidney, liver, lung and intestine were collected at $1 \mathrm{~h}$ post-oral administration. The same doses used for plasma concentration measurement were orally administered to each group of three mice. $100 \mathrm{mg}$ of each tissue were homogenized with the mixture of $3 \mathrm{~mL} 10 \mathrm{mM}$ EDTA, $50 \mathrm{mM} \mathrm{NaClO}$ and $0.1 \%$ $\mathrm{H}_{3} \mathrm{PO}_{4}$ buffer, followed by addition of $0.5 \%$ metaphosphoric acid for protein precipitation. The samples were vortexed for $20 \mathrm{sec}$, centrifuged at $10000 \mathrm{rpm}$ at $4^{\circ} \mathrm{C}$ and then analyzed by HPLC as described above.

2.7. Acute Toxicity. Acute oral toxicity of free GSH, GSHMMT hybrid, and AEA-GSH-MMT hybrid was evaluated according to the OECD guideline 423. Prior to administration, food was withheld for $4 \mathrm{~h}$, but water was available ad libitum. Three mice were orally administrated with four different concentrations of the samples $(5,50,300$, and $2000 \mathrm{mg} / \mathrm{kg}$ ). Group of three mice, receiving identical volume of $0.9 \%$ saline, was served as a control. During 14 days, body weight change, symptoms, and mortality of mice treated with free GSH, GSH-MMT hybrid, or AEA-GSHMMT hybrid were daily recorded.

2.8. Statistical Analysis. The data were expressed as means \pm standard deviation. For statistical analysis, the experimental values were compared with their corresponding control ones. A one-way analysis of variance (ANOVA) in SAS software (Tukey's test, version 11.0) was used to illustrate the significant difference between the experimental group and the control. The statistical significance for all tests was set at $P<0.05$. 


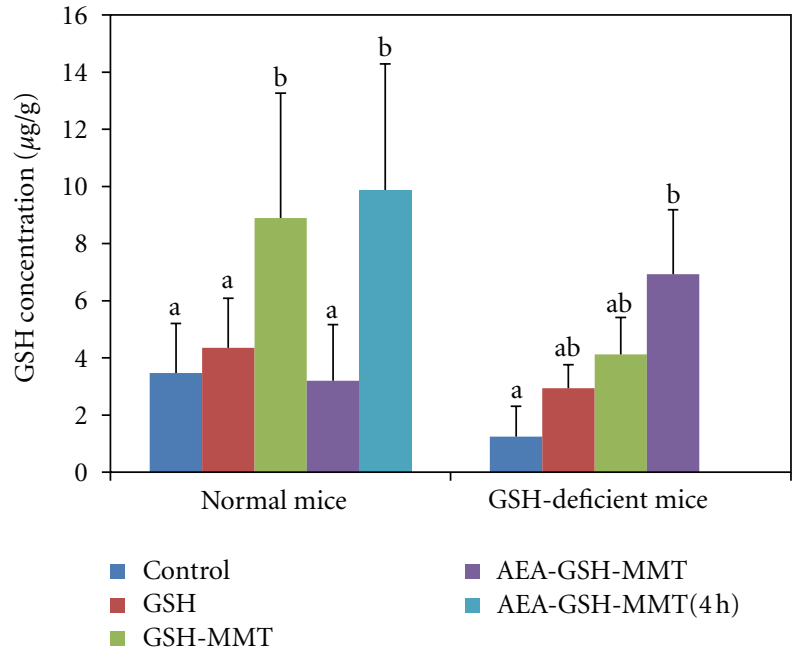

(A)

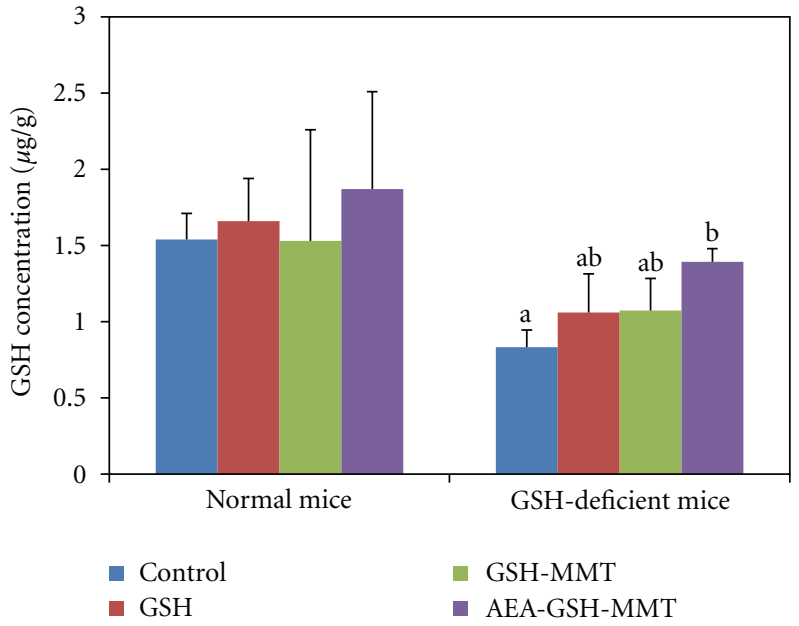

(B)

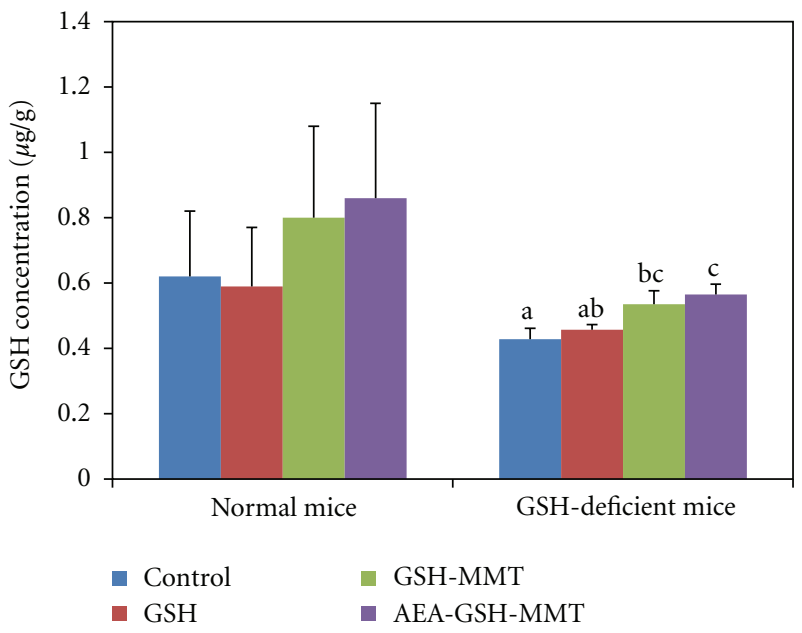

(C)

Figure 3: GSH concentration in the liver (A), heart (B), and kidney (C) at $1 \mathrm{~h}$ postoral administration of free GSH, GSH-MMT hybrid, or AEA-GSH-MMT hybrid in normal as well as GSH-deficient mice. Different letters indicate a significant difference based on ANOVA and the Tukey's test $(P<0.05)$.

\section{Results and Discussions}

3.1. Characterization. In order to synthesize GSH-MMT hybrid systems, we attempted to intercalate protonated GSH into the negatively charged $\mathrm{Na}^{+}-\mathrm{MMT}$ layers at $\mathrm{pH} 2.0$ by cation exchange reaction and optimized the synthetic condition by adding variouss ratio of GSH in comparison with MMT. Figure 1(A) showed the PXRD pattern of MMT and GSH-MMT hybrids, synthesized with 1- (CEC1-), 2- (CEC2) and 3- (CEC3-) fold of CEC. The characteristic $2 \theta$ peaks for MMT, GSH-MMT hybrid (CEC1), GSH-MMT hybrid (CEC2), and GSH-MMT hybrid (CEC3) were found at $7.04^{\circ}$, $5.40^{\circ}, 4.34^{\circ}$, and $4.24^{\circ}$, and $d$-spacing were calculated to be $12.54 \AA, 16.35 \AA, 20.34 \AA$ and $20.82 \AA$, respectively, upon molecular size of interlayer GSH according to the Bragg's law. The peak shifting from higher diffraction angle to lower one is resulted from increased the $d$-spacing, indicating successful interaction of GSH into the interlayer spaces of MMT. In addition, the $2 \theta$ peak was not further shifted to lower one, and d-spacing did not remarkably increase even when CEC3 was added, suggesting that 2-fold of CEC was optimum to synthesize the hybrid system. Schematic diagram for pristine MMT and GSH-MMT hybrid showed that GSH was expected to be intercalated into MMT layers in a monomer form and stabilized by electrostatic interaction, on the basis of XRD pattern (Figure 1(A)). The hybrid was also coated with a basic polymer, AEA, for better stability in vivo, because positively charged AEA polymer under synthetic condition can further stabilize the hybrid by electrostatic interaction. SEM images of the hybrids showed different morphology between GSH-MMT and AEA-GSH-MMT hybrid systems (Figure 1(B)). The particle size of GSH-MMT hybrid was determined to be about $1 \mu \mathrm{m}$, probably resulted from the size of MMT itself [20]. On the other hand, the particle size of GSH-MMT hybrid further increased after polymer coating as shown in Figure $1(\mathrm{~B})(\mathrm{b})$. This result suggests that 


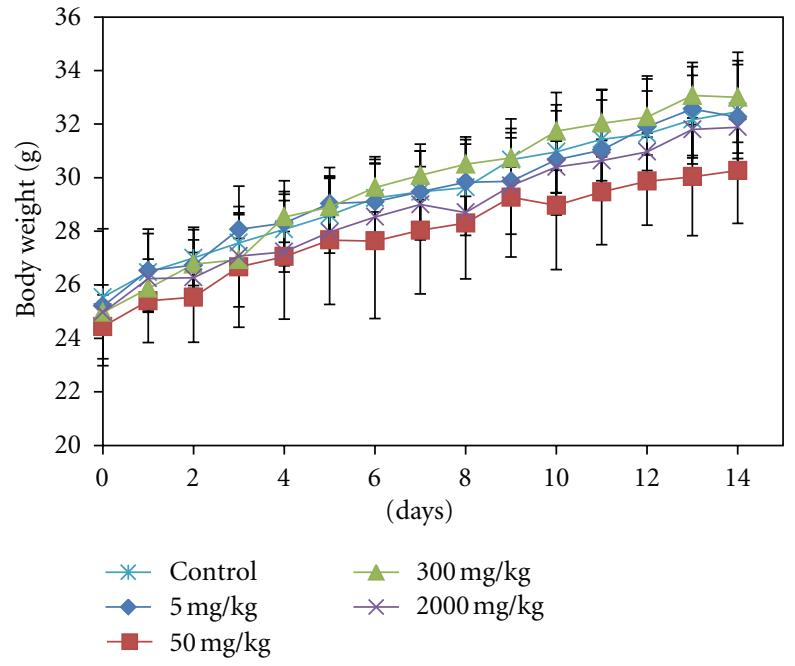

(a)

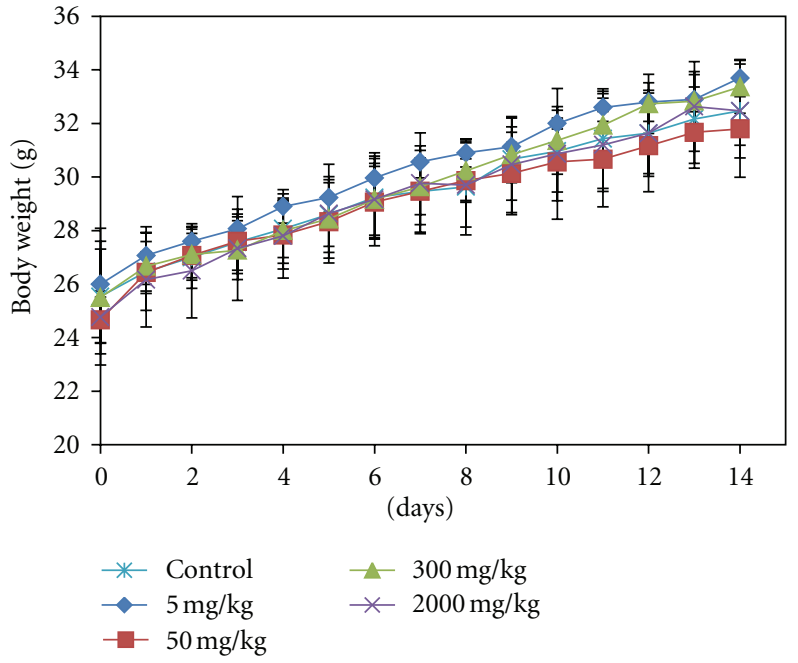

(b)

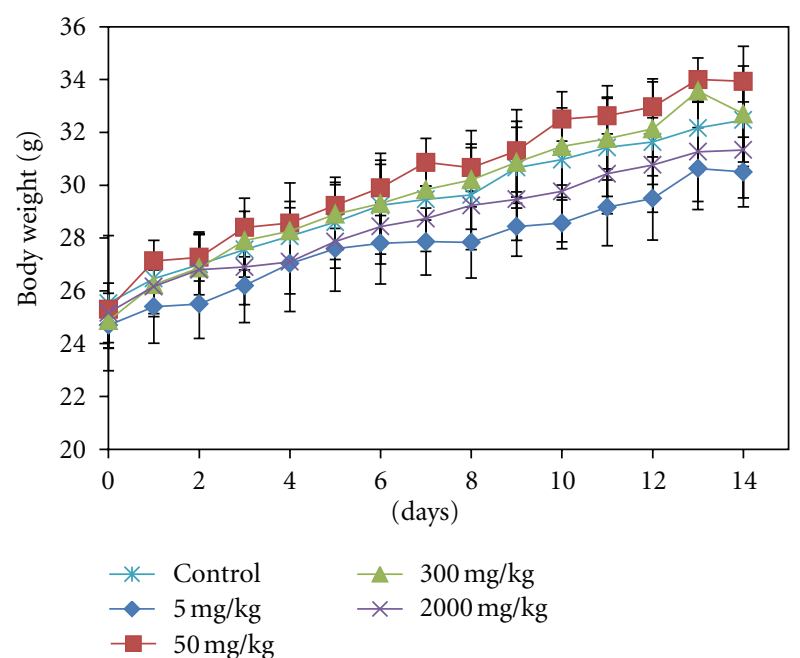

(c)

FIGURE 4: Change in body weight in mice treated with different doses of GSH (a), GSH-MMT hybrid (b), or AEA-GSH-MMT hybrid (c) during 14 days. Single-dose administration was performed by oral gavage. All treated groups showed statistically no significant differences from the control group $(P<0.05)$.

polymer AEA coating of GSH-MMT hybrid was successfully and uniformly performed.

3.2. Plasma Concentration. To evaluate absorption efficiency of both hybrid systems in GSH-deficient mouse model, mice were pretreated with the GSH synthesis inhibitor, L-BSO, via drinking water for 5 days. BSO is a wellknown inhibitor for GSH synthesis by inhibiting specifically and irreversibly $\gamma$-glutamyl-cysteine synthetase $(\gamma$-GCS), the rate-limiting enzyme involved in GSH synthesis process [21]. After $1 \mathrm{~h}$ oral administration of free GSH and GSH-MMT hybrids in normal and GSH-deficient mice, respectively, the plasma samples were analyzed, since our previous study demonstrated that GSH concentration reached the highest level at $1 \mathrm{~h}$ after administration of the hybrids [17]. Figure 2 clearly demonstrated that GSH concentration in the plasma significantly decreased in GSH-deficient control mouse model compared with normal control group, confirming successful inhibition of GSH synthesis. Both hybrids significantly increased GSH level in the plasma in normal as well as in GSH-deficient mice. Interestingly, AEA-GSHMMT hybrid greatly increased the plasma GSH level under both conditions (4.69-fold and 4.75-fold in normal and deficient mice, resp.), suggesting high absorption efficiency of the hybrid system. It is likely that high efficacy of AEAGSH-MMT hybrid system in vivo is closely related to its high structural stability resulted from AEA coating.

3.3. Tissue Distribution. Several tissues such as brain, heart, kidney, liver, lung, and intestine were also collected after oral administration of the hybrids in normal as well as GSH-deficient mice to evaluate biodistribution and delivery 
efficiency of the hybrids to organs. Figure 3 showed that GSH level significantly increased only in the liver when both hybrids were orally administered under normal condition. It is worth noting here that AEA-GSH-MMT hybrid increased GSH level in the liver after $4 \mathrm{~h}$ in comparison with GSHMMT hybrid at $1 \mathrm{~h}$ after treatment (Figure 3(A)), which can be explained by controlled release property of the former due to AEA coating. Lower GSH level in GSH-deficient control mice than that in normal mice was observed in all the tissues, confirming successful induction of the deficient model. Whereas, GSH concentration remarkably increased in the liver, heart, and kidney in GSH-deficient mice, especially receiving AEA-GSH-MMT hybrid. Any significant difference in GSH level between treated and untreated control group was not found in the other organs (data not shown). This result clearly suggests that the hybrid systems enhanced GSH delivery efficiency to various organs and can be more effective under GSH-deficient condition. Therefore, we can expect enhanced pharmaceutical effect of the hybrid systems compared with free GSH intake alone.

3.4. Acute Toxicity. To ensure safety aspect of the hybrid systems and provide critical information about their toxicity potential, the acute oral toxicity of free GSH and GSH-MMT hybrids was evaluated in mice after single dose administration of four different doses (5, 50, 300, and $2000 \mathrm{mg} / \mathrm{kg}$ ). Any remarkable abnormal behaviors, symptoms, and body weight loss were not observed in mice treated with free GSH or GSH-MMT hybrids during 14 days after administration (Figure 4). The $\mathrm{LD}_{50}$ values of free GSH and GSH-MMT hybrids, were estimated to be more than $2000 \mathrm{mg} / \mathrm{kg}$ on the basis of the fact that no mortality was found in all the mice treated with different doses. This result is in good agreement with our previous result, showing low acute oral toxicity of MMT delivery carrier in vivo [16]. Now, we are evaluating the morphological and histopathological changes to ascertain their toxicity potential.

\section{Conclusion}

In this study, we have successfully intercalated a powerful antioxidant, GSH into the interlayer spaces of MMT and obtained two different hybrid systems, GSH-MMT hybrid and AEA-GSH-MMT hybrid, respectively. The synthetic condition was further optimized by using different ratios of GSH compared with MMT. Both GSH-MMT and AEAGSH-MMT hybrids significantly enhanced the absorption amount of GSH under both normal and GSH-deficient conditions as indicated by increased plasma GSH concentration. Tissue distribution study demonstrated that both hybrids increased GSH level only in the liver in normal mice, while high GSH concentration was found in the liver, heart, and kidney in GSH-deficient mouse model. Delivery efficiency of GSH to organs was more effective when AEAGSH-MMT hybrid was orally administered, especially under GSH-deficient condition. Moreover, both the hybrid systems do not seem to induce acute oral toxicity. Therefore, all the results suggest that GSH-MMT hybrid systems will be promising candidates to enhance absorption and delivery efficiency of oral GSH, providing new insight into their pharmaceutical application in GSH-deficient patients.

\section{Acknowledgments}

This paper was supported by Basic Science Research Program through the National Research Foundation of Korea (NRF) funded by the Ministry of Education, Science and Technology (2011-0003755), and partly by National Research Foundation of Korea Grant funded by the Korean Government (SRC Program: 2012-0000650).

\section{References}

[1] H. Sies, "Glutathione and its role in cellular functions," Free Radical Biology and Medicine, vol. 27, no. 9-10, pp. 916-921, 1999.

[2] E. Brzezinska-Ślebodzinska, A. B. Ślebodzinski, B. Pietras, and G. Wieczorek, "Antioxidant effect of vitamin E and glutathione on lipid peroxidation in boar semen plasma," Biological Trace Element Research, vol. 47, no. 1-3, pp. 69-74, 1995.

[3] L. Milne, P. Nicotera, S. Orrenius, and M. J. Burkitt, "Effects of glutathione and chelating agents on copper-mediated DNA oxidation: pro-oxidant and antioxidant properties of glutathione," Archives of Biochemistry and Biophysics, vol. 304, no. 1, pp. 102-109, 1993.

[4] P. Li, Y. L. Yin, D. Li, W. S. Kim, and G. Wu, "Amino acids and immune function," British Journal of Nutrition, vol. 98, no. 2, pp. 237-252, 2007.

[5] I. A. Cotgreave and R. G. Gerdes, "Recent trends in glutathione biochemistry-glutathione-protein interactions: a molecular link between oxidative stress and cell proliferation?" Biochemical and Biophysical Research Communications, vol. 242, no. 1, pp. 1-9, 1998.

[6] W. Dröge, V. Hack, R. Breitkreutz et al., "Role of cysteine and glutathione in signal transduction, immunopathology and cachexia," BioFactors, vol. 8, no. 1-2, pp. 97-102, 1998.

[7] S. Gerald, S. Joel, T. Diane, and R. C. Susan, "The effectiveness of a mixture of $\beta$-carotene, $\alpha$-tocopherol, glutathione, and ascorbic acid for cancer prevention," Nutrition and Cancer, vol. 20, no. 2, pp. 145-151, 1993.

[8] L. A. Herzenberg, S. C. De Rosa, J. G. Dubs et al., "Glutathione deficiency is associated with impaired survival in HIV disease," Proceedings of the National Academy of Sciences of the United States of America, vol. 94, no. 5, pp. 1967-1972, 1997.

[9] J. D. Peterson, L. A. Herzenberg, K. Vasquez, and C. Waltenbaugh, "Glutathione levels in antigen-presenting cells modulate Th1 versus Th2 response patterns," Proceedings of the National Academy of Sciences of the United States of America, vol. 95, no. 6, pp. 3071-3076, 1998.

[10] F. Jahoor, A. Jackson, B. Gazzard et al., "Erythrocyte glutathione deficiency in symptom-free HIV infection is associated with decreased synthesis rate," American Journal of Physiology, vol. 276, no. 1, pp. E205-E211, 1999.

[11] T. M. Hagen, G. T. Wierzbicka, B. B. Bowman, T. Y. Aw, and D. P. Jones, "Fate of dietary glutathione: disposition in the gastrointestinal tract," American Journal of Physiology, vol. 259, no. 4, pp. G530-G535, 1990.

[12] A. Witschi, S. Reddy, B. Stofer, and B. H. Lauterburg, "The systemic availability of oral glutathione," European Journal of Clinical Pharmacology, vol. 43, no. 6, pp. 667-669, 1992. 
[13] M. E. Anderson, R. J. Bridges, and A. Meister, "Direct evidence for inter-organ transport of glutathione and that the non-filtration renal mechanism for glutathione utilization involves $\gamma$-glutamyl transpeptidase," Biochemical and Biophysical Research Communications, vol. 96, no. 2, pp. 848-853, 1980.

[14] T. Y. Aw, "Intestinal glutathione: determinant of mucosal peroxide transport, metabolism, and oxidative susceptibility," Toxicology and Applied Pharmacology, vol. 204, no. 3, pp. 320328, 2005.

[15] A. Fudala, I. Palinko, and I.s Kiricsi, "Preparation and characterization of hybrid organic-inorganic composite materials using the amphoteric property of amino acids: amino acid intercalated layered double hydroxide and montmorillonite," Inorganic Chemistry, vol. 38, no. 21, pp. 4653-4658, 1999.

[16] M. Baek, J. A. Lee, and S. J. Choi, "Toxicological effects of cationic clays in vitro and in vivo," Molecular \& Cellular Toxicolgy, vol. 8, no. 1, pp. 95-101, 2012.

[17] M. Baek, J. H. Choi, and S. J. Choi, "Montmorillonite intercalated with glutathione for antioxidant delivery: synthesis, characterization, and bioavailability evaluation," International Journal of Pharmaceutics, vol. 425, no. 1-2, pp. 29-34, 2012.

[18] T. Y. Aw, G. Wierzbicka, and D. P. Jones, "Oral glutathione increases tissue glutathione in vivo," Chemico-Biological Interactions, vol. 80, no. 1, pp. 89-97, 1991.

[19] Ö. Yilmaz, S. Keser, M. Tuzcu et al., "A practical HPLC method to measure reduced (GSH) and oxidized (GSSG) glutathione concentrations in animal tissues," Journal of Animal and Veterinary Advances, vol. 8, no. 2, pp. 343-347, 2009.

[20] A. Cadene, S. Durand-Vidal, P. Turq, and J. Brendle, "Study of individual Na-montmorillonite particles size, morphology, and apparent charge," Journal of Colloid and Interface Science, vol. 285, no. 2, pp. 719-730, 2005.

[21] T. Watanabe, H. Sagisaka, S. Arakawa et al., "A novel model of continuous depletion of glutathione in mice treated with L-Buthionine (S,R)-sulfoximine," Journal of Toxicological Sciences, vol. 28, no. 5, pp. 455-469, 2003. 

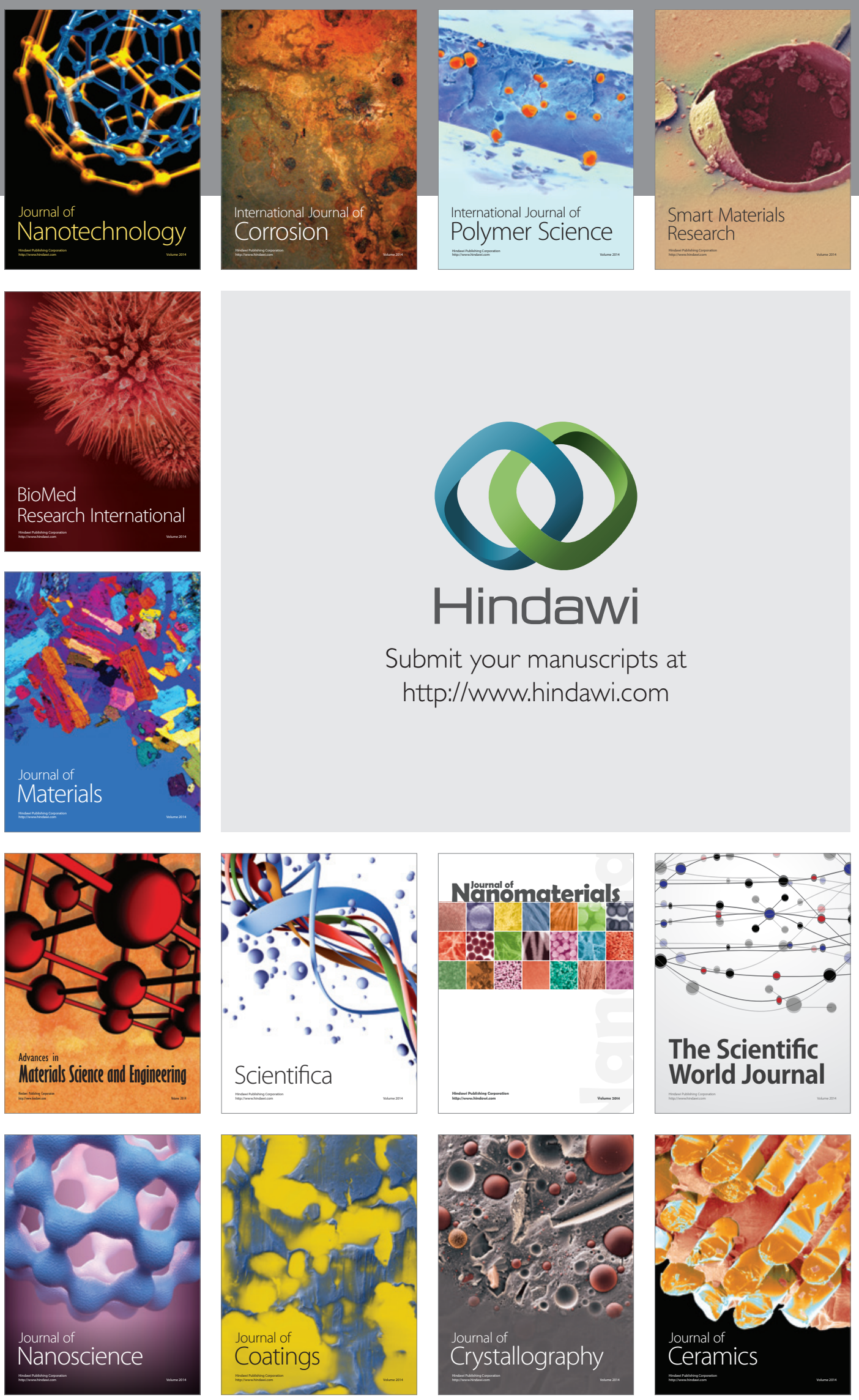

The Scientific World Journal

Submit your manuscripts at

http://www.hindawi.com

\section{World Journal}

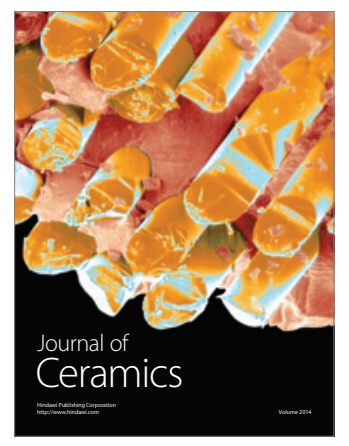

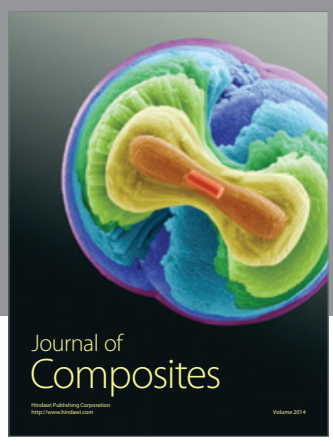
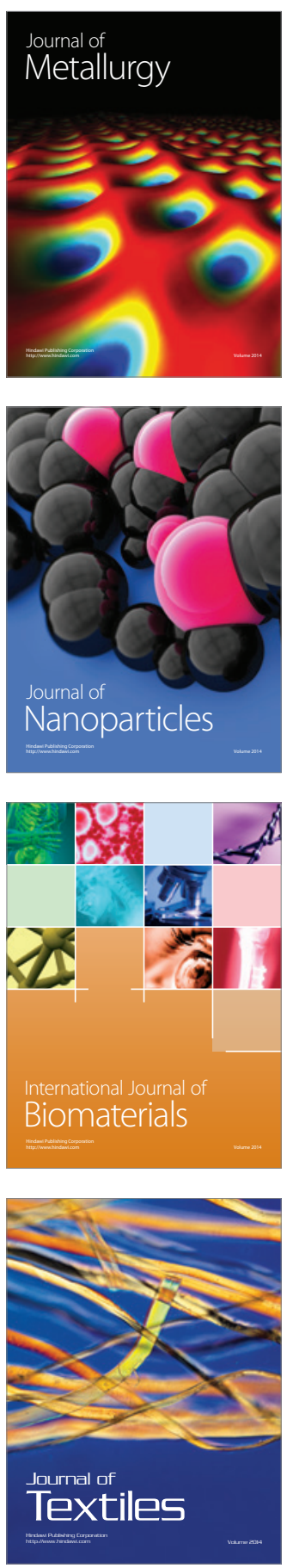